\title{
Glucose Metabolism Characteristics of Extra-Hypothalamic Cortex in Patients With Hypothalamic Hamartomas (HH) Undergoing Epilepsy Evaluation: A Retrospective Study of 16 Cases
}

Yan-Feng Yang ${ }^{1}$, Peng-Hu Wei ${ }^{1}$, Fei Meng ${ }^{1}$, Yang An ${ }^{1}$, Xiao-Tong Fan ${ }^{1}$, Yi-He Wang ${ }^{1}$, Di Wang ${ }^{2}$, Lian-Kun Ren ${ }^{2}$, Yong-Zhi Shan ${ }^{1 *}$ and Guo-Guang Zhao ${ }^{1 *}$

${ }^{1}$ Department of Neurosurgery, Xuan Wu Hospital, Capital Medical University, Beijing, China, ${ }^{2}$ Department of Neurology, Xuan Wu Hospital, Capital Medical University, Beijing, China

OPEN ACCESS

Edited by:

Luiz Eduardo Betting, São Paulo State University, Brazil

Reviewed by:

Barbara Juarez Amorim, Campinas State University, Brazil Csaba Juhasz,

Wayne State University, United States

${ }^{*}$ Correspondence: Yong-Zhi Shan

shanyongzhi@xwhosp.org

Guo-Guang Zhao

ggzhao@vip.sina.com

Specialty section: This article was submitted to

Epilepsy,

a section of the journal

Frontiers in Neurology

Received: 27 July 2020 Accepted: 09 December 2020 Published: 14 January 2021

Citation:

Yang Y-F, Wei P-H, Meng F, An Y, Fan $X-T$, Wang $Y-H$, Wang $D$, Ren $L-K$,

Shan Y-Z and Zhao G-G (2021) Glucose Metabolism Characteristics of Extra-Hypothalamic Cortex in Patients With Hypothalamic Hamartomas $(H H)$

Undergoing Epilepsy Evaluation: A Retrospective Study of 16 Cases. Front. Neurol. 11:587622. doi: 10.3389/fneur.2020.587622
Purpose: There are few studies on the glucose metabolic characteristics of the extra-hypothalamic cortex in the hypothalamic hamartomas $(\mathrm{HH})$. A comprehensive understanding of pathogenic progression of the disease is required from the perspective of cortical metabolism; therefore, we aimed to characterize metabolic characteristics of extra-hypothalamic in $\mathrm{HH}$ patients.

Methods: We investigated the metabolic characteristics of $16 \mathrm{HH}$ patients, all of whom underwent epilepsy evaluation at Xuan Wu Hospital between 2017 and 2019. The lateralization and cortical distribution pattern of hypometabolism was assessed and related to $\mathrm{HH}$ mass neuroanatomy on magnetic resonance imaging (MRI) as well as scalp-electroencephalogram (scalp-EEG) abnormalities. Furthermore, asymmetry measurements of region of interest $(\mathrm{ROI})$ in the temporal cortex (hippocampal formation, amygdala, and lateral temporal neocortex) were quantitatively assessed based on the normalized average positron emission tomography (PET) voxel values. The surgery prognosis was assessed using the International League Against Epilepsy (ILAE) classification system.

Results: The lateralization of hypometabolism in global visual ratings was consistent with the $\mathrm{HH}$ mass lateralization seen on MRI. Cortical hypometabolism showed three patterns depending whether the $\mathrm{HH}$ mass involved mammillary bodies, middle hypothalamus nucleus, or both. The three patterns were hypometabolism of the mesial temporal cortex with symptom of mesial temporal epilepsy (3/16, pattern I), lateral temporal, and extratemporal (frontal or parietal) cortex with symptom of neocortex temporal or frontal epilepsy (5/16, pattern II), and mesial and lateral temporal cortex and extratemporal (frontal or parietal) cortex with varied symptoms (8/16, pattern III), respectively. A significant difference in PET voxel values was found between bilateral hippocampal formation $(P=0.001)$ and lateral temporal neocortex in the third group $(P=0.005)$. We suggest that the hypometabolic characteristics of the extra-hypothalamic cortex in 
$\mathrm{HH}$ patients have three patterns. The final cortical hypometabolic pattern depends on the neuroanatomic location of the $\mathrm{HH}$ mass and was consistent with the main involved cortex of the interictal and ictal discharges. The third hypometabolic pattern with the most extensive cortical hypometabolism has a poorer prognosis.

Keywords: hypothalamic hamartomas, epilepsy, extra-hypothalamic cortex, PET, glucose metabolism

\section{INTRODUCTION}

Hypothalamic hamartomas $(\mathrm{HH})$ are characterized by a variety of clinical spectrum, evolving from disease with epilepsy, central precocious puberty, or cognitive and behavioral impairment to catastrophic epileptic encephalopathy (1-6). The discovery of intrinsic epileptogenicity caused by $\mathrm{HH}$ has been confirmed by electrophysiology and histopathologic characterization of $\mathrm{HH}$ neurons (7-11). However, various surgical interventions targeting $\mathrm{HH}$ do not abate seizures, and the electrophysiological evidence in ineffective cases suggests that distant cortical regions are involved, especially the temporal and frontal cortex (12). This progressive epileptogenic encephalopathy suggests that the evolution of disease may correlate with abnormal cortical network external to the HH. This is consistent with the network hypothesis of focal epilepsy (13) that the epileptogenic zone is not only limited to focal lesion (HH mass) but may also involve the distant cerebral cortex external to the HH mass. Studies have confirmed that gelastic seizures are correlated with abnormal neural activation within the $\mathrm{HH}(10)$ and other seizure types are related to neural activation involved with cortical regions (14). These findings suggest that an independent, third-stage kindling like secondary epileptogenesis may exist in patients with $\mathrm{HH}$ $(2,15)$.

Most of the above evidence is derived from electrophysiological studies, limited by lack of spatial resolution; the lobar distribution characteristics of the cortex external to $\mathrm{HH}$ are not well-studied. The rapid development of imaging, functional neuroimaging techniques such as single-photon emission computed tomography (SPECT), and $\left[{ }^{18} \mathrm{~F}\right]$ fluorodesoxyglucose positron emission tomography $\left[\left[{ }^{18} \mathrm{~F}\right]\right.$ FDG-PET] provides additional, independent information for structural neuroimaging. The SPECT and PET tracers can measure regional cerebral blood flow and glucose metabolism associated with epileptic dysfunction. Therefore, they can be used as surrogate markers of the functional deficit cortex (16).

One SPECT study found two hyperperfusion patterns in the cortex of HH-related epilepsy (17). One pattern showed ipsilateral temporal hyperfusion and its clinical and electroencephalography (EEG) features resembled temporal lobe epilepsy. This may suggest a lateral spread pattern to the temporal lobe that determines a focal "pseudo-temporal" epilepsy. The

\footnotetext{
Abbreviations: $\mathrm{HH}$, hypothalamic hamartomas; $\left[{ }^{18} \mathrm{~F}\right]$ FDG-PET, $\left[{ }^{18} \mathrm{~F}\right]-$ fluorodesoxyglucose positron emission tomography; SPECT, single-photon emission computed tomography; EEG, electroencephalography; MRI, magnetic resonance imaging; MPRAGE, magnetization-prepared rapid-gradient echo; ROS, region of interest; IQR, interquartile range; ILAE, International League Against Epilepsy.
}

other pattern showed ipsilateral frontal hyperfusion, and its clinical and EEG showed symptomatic generalized epilepsy with a more catastrophic syndrome. It was possible that a vertical spread pattern involved deep midline structure, such as the medial frontal cortex. Patients with catastrophic syndrome are also often accompanied by a decline in cognitive function (18, 19). Wagner et al. (6) showed in the study that regions of reduced glucose metabolism in cognitively impaired patients mainly were in the frontal and parietal cortex.

PET studies show higher sensitivity and specificity in the localization of the functional deficit cortex than SPECT studies (20). In a PET study of $\mathrm{HH}$, the hypometabolic extrahypothalamic cortex, like that of electroclinical findings, varied greatly between patients and was related to most seizure types except gelastic seizure (21). The hypometabolic extrahypothalamic cortex includes the temporal, frontal, and parietal cortex. In addition, Parvizi et al. (22) showed that the specific anatomical location of the $\mathrm{HH}$ mass in patients may determine the possible involvement of specific hypothalamic nuclei and neuroanatomical routes of seizure propagation in these cases. Studies showed $75 \%$ of patients with other seizure types more commonly develop focal seizures with frontal or temporal onset $(23,24)$. This may depend on the tight connections of $\mathrm{HH}$ through mammillary bodies (temporal lobe) or to the middle hypothalamic nucleus (frontal lobe) (25). It may suggest that there are hypothalamic candidate nuclei in the seizure propagation in patients with $\mathrm{HH}$. $\mathrm{HH}$ mass may make use of the adjacent normal hypothalamic nuclei for propagation of epileptic discharges and form their own abnormal connections with extrahypothalamic structure leading to cortical hypometabolism (22). However, little research has focused on the cortical distribution characteristics of glucose metabolism of extra-hypothalamic cortex. In this paper, we aim to investigate the abnormality of extra-hypothalamic cortex from the perspective of cortical metabolism in patients with $\mathrm{HH}$ and further to find whether the cortical distribution characteristics are correlated with the neuroanatomical location of $\mathrm{HH}$ mass and scalp-EEG findings.

\section{METHODS}

\section{Patients}

The present retrospective study included 16 patients (nine males, seven females) with medically intractable epilepsy due to $\mathrm{HH}$, all of whom underwent epilepsy evaluation, and thermocoagulation was performed at Xuan Wu Hospital between 2017 and 2019. We excluded cases with (a) previous history of surgical intervention and (b) any brain abnormalities other than $\mathrm{HH}$ in image. $\left[{ }^{18} \mathrm{~F}\right]$ FDG-PET scans were acquired in patients with multiple seizure 
types other than gelastic seizure or with severe cognitive or behavioral disorders, completely in accordance with clinical needs. In addition, EEG and MRI were acquired in the context of presurgical evaluation and were available for retrospective analysis. The clinical characteristics of the included patients are shown in Table 1. The mean age of patients was $11.75 \pm 10.31$ years (range 2-37 years). The mean age at seizure onset was $3.73 \pm 4.08$ (range $0.08-16$ years). The study was approved by the ethics committee of the Capital Medical University, and all included patients and caregivers provided written informed consent to participate and for publication.

\section{MRI Data}

All patients underwent acquisition of a T1-weighted images, T1-magnetization-prepared rapid-gradient echo (MPRAGE) and
T2-weighted images (Siemens 3T). The diagnosis of the hamartoma was based on MRI of the brain, which was isointense to cerebral cortex on T1-weighted images and isointense or hyperintense on T2-weighted images. The classification of $\mathrm{HH}$ was classified according to Delalande and Fohlen's study by two experienced imaging doctors blinded to clinical information (26). The classification is defined as horizontal base of attachment below the normal position of the floor of the third ventricle (class 1), vertical plane of attachment to the wall of the third ventricle, completely above the normal position of the floor of the third ventricle (class 2), the plane of attachment that extends both above and below the normal position of the floor of the third ventricle (class 3), and giant lesions which are bigger than $8 \mathrm{~cm}^{3}$ (class 4 ). The $\mathrm{HH}$ mass lateralization on MRI according to the asymmetric attachment to the

TABLE 1 | Demographic and clinical features of patients $(n=16)$.

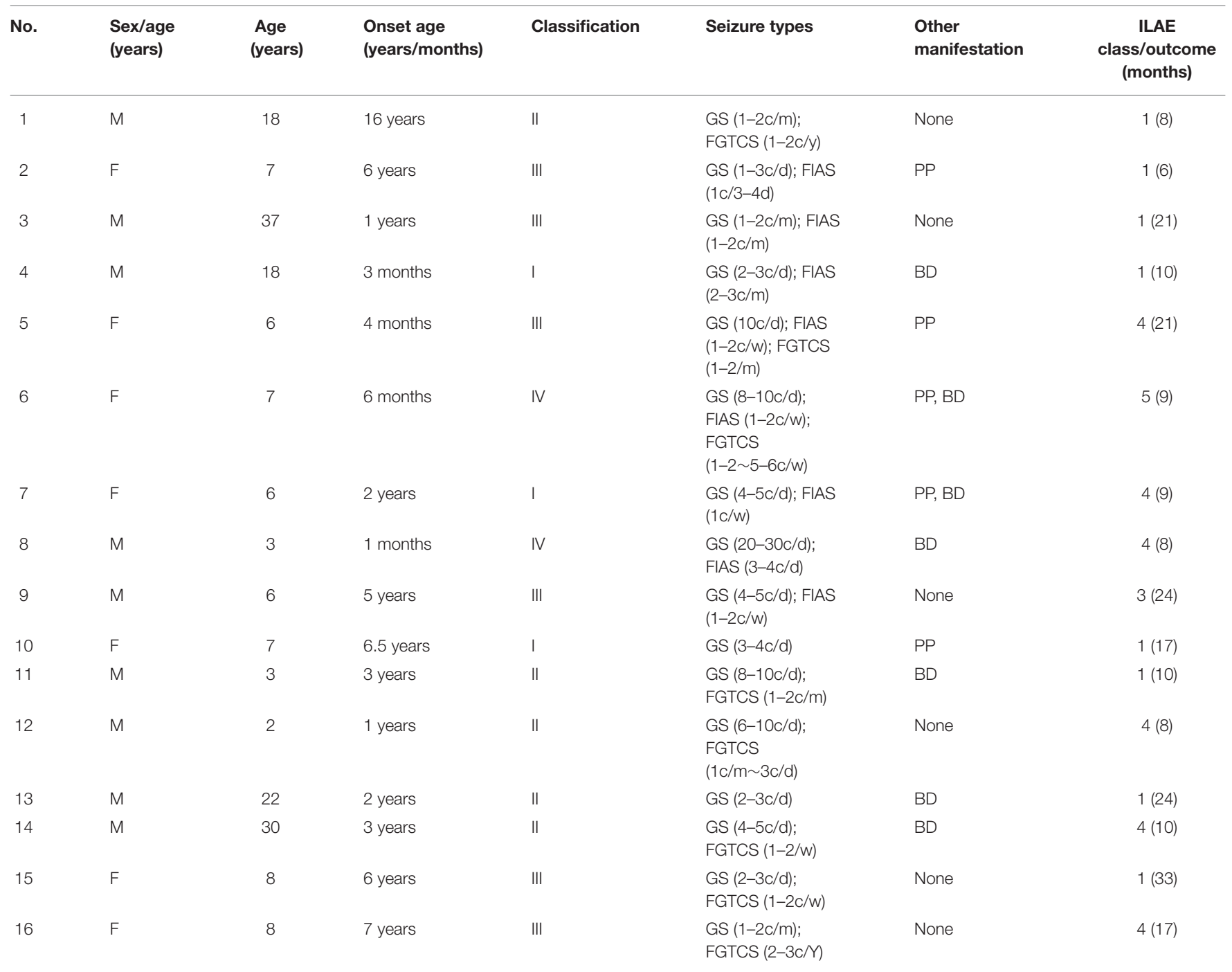

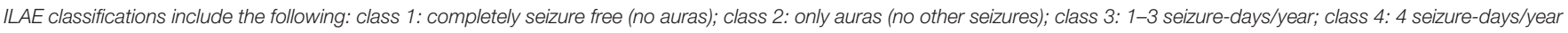
to a decrease of $50 \%$ from pretreatment seizure frequency; class 5: from 50\% decrease up to $100 \%$ increase; class 6: $\geq 100 \%$ increase in seizure frequency.

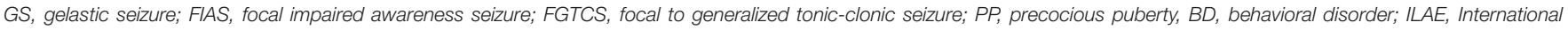
League Against Epilepsy. 
hypothalamus proper with description of this by category: (1) The hamartoma connection to the middle (middle hypothalamic right (right-sided and predominantly right-sided); (2) left (leftnuclei) and posterior segments (mammillary bodies) of the sided and predominantly left-sided); and (3) not lateralized.

hypothalamus was determined. Involvement of the mammillary

TABLE 2 | PET, MRI, and scalp-EEG characteristics of $\mathrm{HH}$ patients.

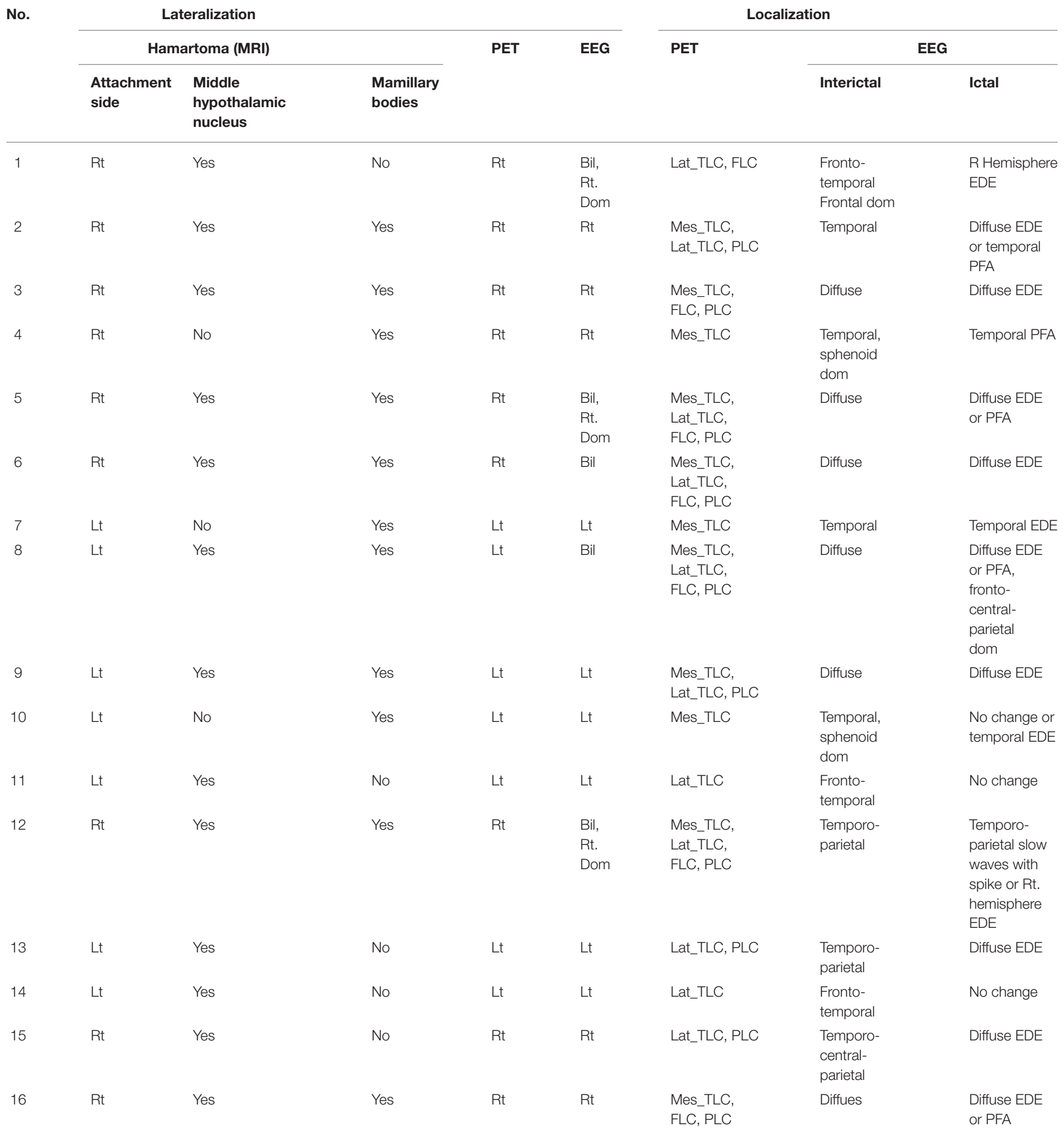

Bil, bilateral; Lt, left; Rt, right; Dom, dominant; Lat, lateral; Mes, mesial; TLC, temporal lobe cortex; FLC, frontal lobe cortex; PLC, parietal lobe cortex; EDE, electrodecremental events; PFA, paroxysmal fast activity. 
bodies was accepted when the T1-weighted images showed there was an interruption on the continuity of the hypersignal in surrounding these structures which were in contact with the $\mathrm{HH}$ mass. This criterion was important because the structure of the mammillary bodies remained unchanged even if there was a significant shift in some patients (25).

\section{Analysis of Long-Term Scalp-EEG Monitoring and Clinical Semiology}

Prolonged EEG data were acquired via video-EEG monitoring (Micromed, Italy). The recording parameters were as follows: sampling rate $=512 \mathrm{~Hz}$, low filter setting $=0.16 \mathrm{~Hz}$, and high filter setting $=70 \mathrm{~Hz}$. Each EEG sample was analyzed and classified according to the following criteria: (a) lateralization: (i) left, (ii) right, or (iii) bilateral; (b) localization: (i) frontal, (ii) temporal, (iii) parietal, (iv) occipital, (v) vertex, or (vi) not applicable (NA, when generalized or non-localized); (c) abnormal waveform: (i) background slowing, (ii) spike, (iii) spike and wave complex, (iv) polyspike complex. Three independent clinicians utilized Lüders' semiological seizure classification to evaluate ictal semiology (27).

\section{$\left[{ }^{18}\right.$ F] FDG PET Data}

\section{Acquisition}

PET images were acquired on a United Imaging PET scanner, providing 2.4-mm-thick slices, with an isotropic spatial resolution of $5 \mathrm{~mm}$. Patients were requested to report any seizures on the day of the scan. The scans cannot be performed if patients had a seizure within the last $12 \mathrm{~h}$. Patients were instructed to lie at rest in a semidark room with eyes closed and ears unplugged when a bolus of 5-10 $\mathrm{mCi}$ of FDG was then injected intravenously. After an uptake phase of $60 \mathrm{~min}$, we performed a 30-40-min duration image acquisition. During acquisition, a facemask ensured the stable position of the head in these patients. The resulting PET images were qualitatively reviewed by an experienced nuclear physician, blinded to other clinical information.

\section{Global Visual Ratings and Quantitative Analysis of $\left[{ }^{18} \mathrm{~F}\right]$ FDG PET}

The global visual ratings of the patients' lateralization and cortical distribution of hypometabolism were classified based on the asymmetrical assessment of cerebral cortex which was identified during a multidisciplinary patient management conference at
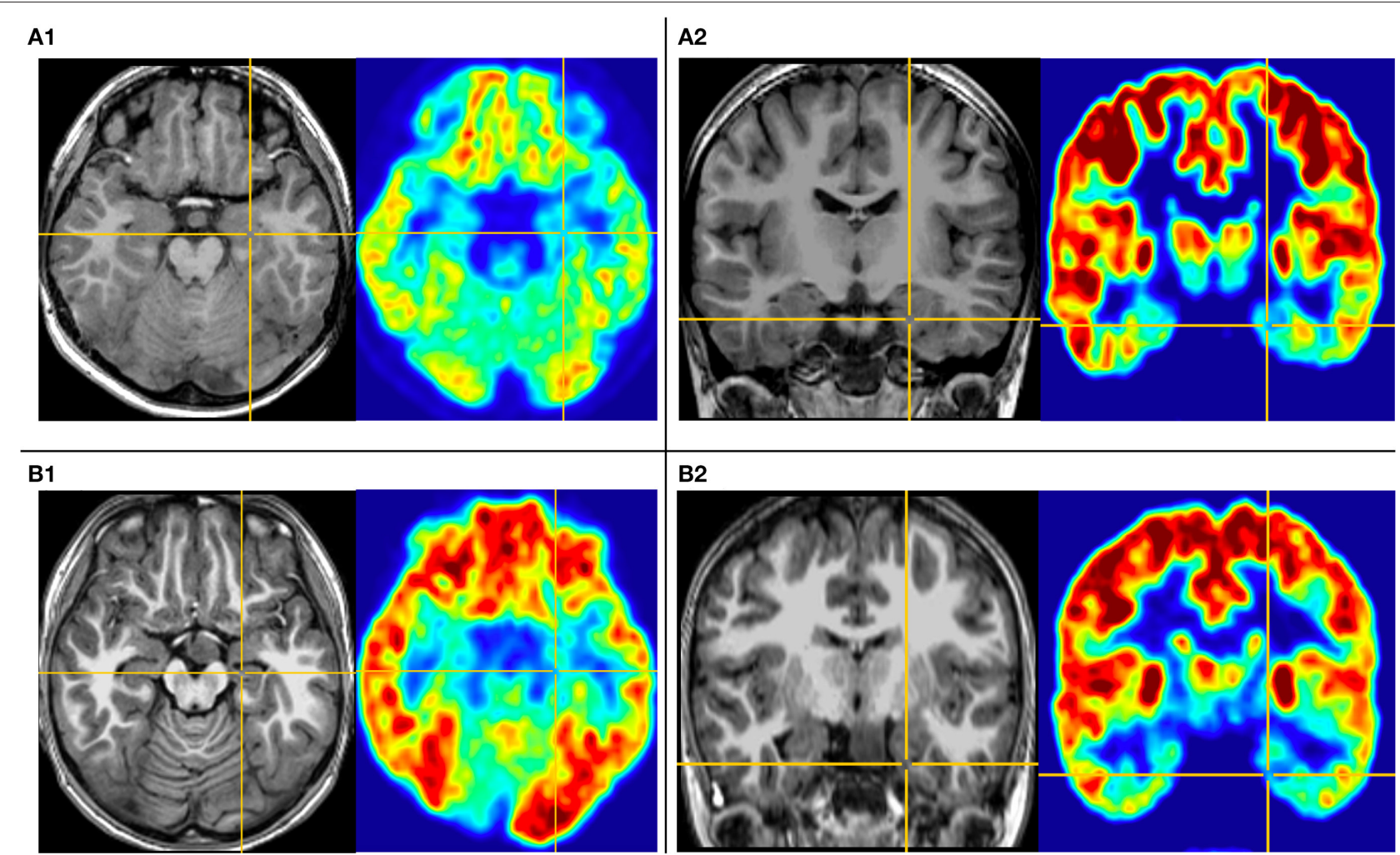

B2

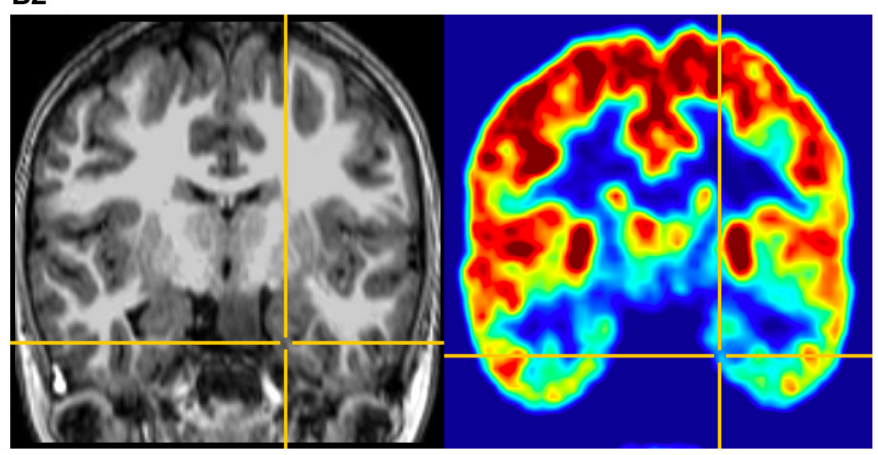

FIGURE 1 | This is the MRI-PET of Pt. 10 (A) and Pt. 7 (B) in the first cortical hypometabolic pattern group. T1-MPRAGE (left) and PET images (right) were co-registered and normalized in MNI reference. (A) Both the horizontal (A1) and the coronal (A2) plane of PET showed that the hypometabolic cortex is located in the left mesial temporal cortex. Quantification analysis of Pt. 10 showed the PET voxel value of the right hippocampus formation of 1.970, and for the left hippocampus formation of 1.771, with an asymmetric value of 11.3\%. (B) Both the horizontal (B1) and the coronal (B2) of PET showed that the hypometabolic cortex is located in the left mesial temporal cortex. Quantification analysis of Pt. 7 showed the PET voxel value of the right hippocampus formation of 1.962, and for the left hippocampus formation of 1.759 , with an asymmetric value of $11.5 \%$. 
the Epilepsy Center. Then the results were checked with the PET report interpreted by an experienced PET physician blinded to clinical information. The standard used to determine hypometabolism on PET reports is an asymmetrical standard uptake value (SUV) of the bilateral cortex of more than $10 \%$. If interpretation differed, a consensus opinion was reached after joint review.

Image analysis was conducted with tools from the FMRIB Software Library (https://fsl.fmrib.ox.ac.uk/fsl/fslwiki/ FslInstallation), Statistical Parametric Mapping software (SPM 12, Wellcome Department of Imaging Neuroscience, www.fil.ion.ucl.ac.uk/spm12) and 3D Slicer (http://www. slicer.org). Brain extraction tools were first used to remove non-brain tissue from structural MR images. Subsequently, T1-MRI and PET images were co-registered and spatial normalization was computed from the SPM12 T1-MRI brain template referenced by the Montreal Neurological Institute (MNI) and applied to all images. The obtained image was smoothed using 3D isotropic Gaussian kernel of $3 \mathrm{~mm}$ in width (28). Three regional masks were utilized to extract PET values for hippocampal formation, amygdala, and lateral temporal cortex. The masks were obtained from the FreeSurfer parcellation map and contained using FreeSurfer (http://surfer.nmr.mgh.harvard.edu). To reduce intersubject variance before performing statistical analysis, the voxel value

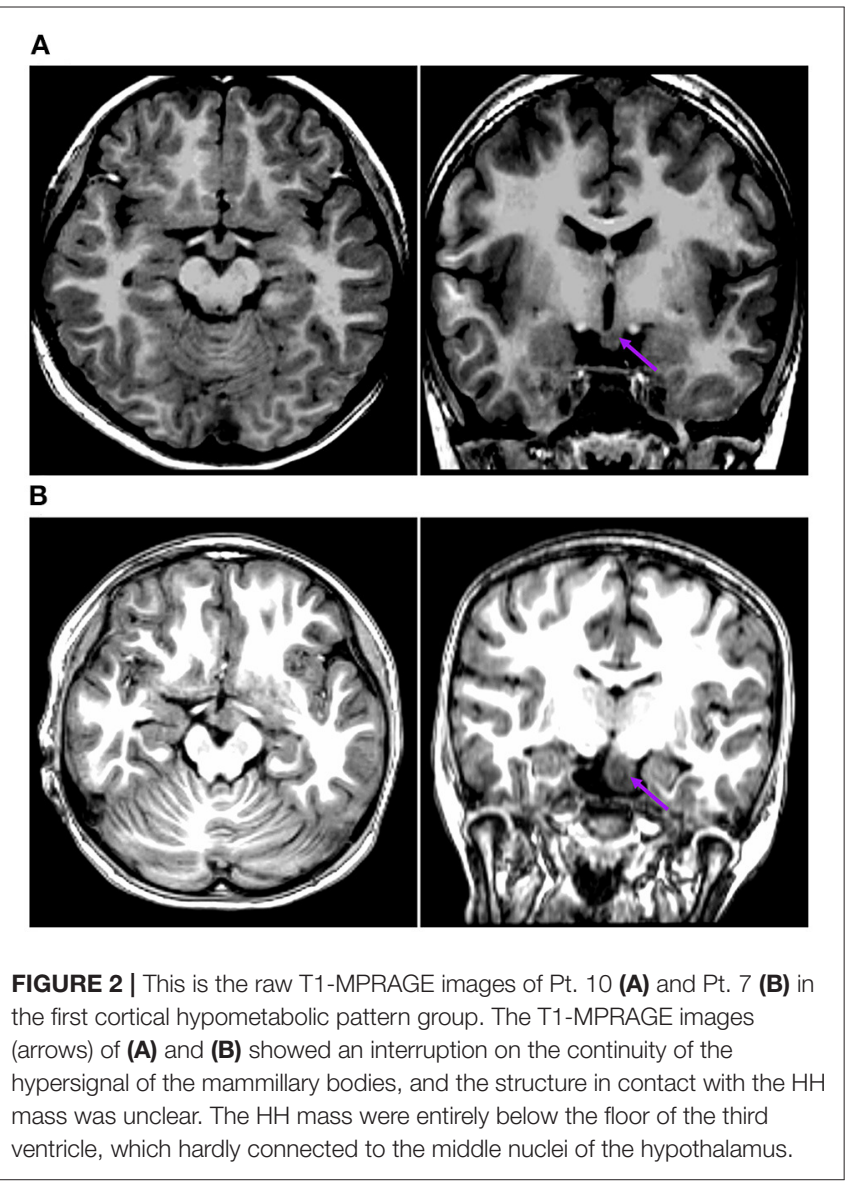

for each patient's PET images was divided by the average voxel value measured in the cerebellum (29). Before doing so, an investigator performed a visual assessment of all images to ensure that there were no metabolic changes in the cerebellum.

In addition, metabolic asymmetric value (MAV) of the ROI of the temporal cortex (hippocampal formation, amygdala, and lateral temporal neocortex) was quantitatively assessed based on the normalized average PET voxel values. The MAV can be calculated with the following formula: MAV $=$ (contralateral value-ipsilateral value)/ipsilateral value. We regarded normalized regional differences $>10 \%$ as asymmetrical (30).

At last, we further analyzed the asymmetry value of the temporal cortex (hippocampal formation, amygdala, and lateral temporal neocortex) of the different cortical hypometabolic pattern groups based on the normalized average PET voxel value.

\section{Prognosis Evaluation}

The surgery prognosis was assessed according to the ILAE (31) classification system through telephone calls and outpatient visits. ILAE outcome classification is an ordinal scale based on seizure-days/year, with categories defined as complete seizure freedom without auras (class 1), only auras without other seizures (class 2), 1-3 seizure-days/year (class 3), 4 seizure-days/year to a decrease of $50 \%$ from pretreatment seizure frequency (class 4), from 50\% decrease up to $100 \%$ increase (class 5), and $\geq 100 \%$ increase in seizure frequency (class 6).

\section{Statistical Analysis}

The normalized average PET values were expressed as median [interquartile range (IQR)] and compared between the contralateral and ipsilateral temporal cortex with the Wilcoxon signed-rank test. Wilcoxon signed-rank tests were calculated with the Statistical Package for the Social Sciences for Windows (Version 22; IBM, Armonk, New York, USA).

\section{RESULTS}

\section{Laterality of HH on MRI and Cortical Glucose Hypometabolism}

$\mathrm{HH}$ lateralization seen on MRI was consistent with the lateralization of reduced glucose metabolism in global visual ratings. In seven of the 16 patients, $\mathrm{HH}$ mass was attached or predominantly attached to the left hypothalamus, and the hypometabolism of the extrahypothalamic cortex was on the left hemisphere. In another nine patients, $\mathrm{HH}$ attached or predominantly attached to the right hypothalamus, causing lateralization of the cortical hypometabolism in the right. For further details, see Table 2. 


\section{Cortical Distribution Pattern of Hypometabolism and Correlation With Neuroanatomy of HH on MRI and Electroclinical Abnormalities}

The extra-hypothalamic cortex in patients with $\mathrm{HH}$ mainly presented three hypometabolic patterns, which were related to the neuroanatomical location of $\mathrm{HH}$ mass and electroclinical abnormalities (see Table 2). In three patients, the HH mass established a connection only to the posterior areas of the hypothalamus (mammillary bodies), in five patients, the connection to the middle areas of the hypothalamus (middle hypothalamic nucleus), and in another eight patients, the connection to both the mammillary bodies and middle hypothalamic nucleus.

The first pattern (pattern I) showed three patients (Pt. 4, 7, and 10) whose cortical hypometabolism was mainly in the limbic cortex of the mesial temporal lobe, including the hippocampal formation, parahippocampal gyrus, or entorhinal cortex. Three patients showed reduced glucose metabolism only in the mesial temporal cortex (Figure 1). The T1-MPRAGE of the three patients showed $\mathrm{HH}$ masses were entirely below the floor of the third ventricle with minimal ventricular displacement. The T1 image showed an interruption on the continuity of the hypersignal surrounding these structures in contact with the $\mathrm{HH}$ mass, which implied the tight connection between $\mathrm{HH}$ mass with the mammillary bodies (Figure 2). Of the three patients with the first hypometabolic pattern, interictal discharge predominantly occurred in the temporal cortex with the sphenoid electrode predominantly (two of three). The ictal EEG showed no change (one of three), temporal paroxysmal fast activity (one of three), or temporal electrodecremental events (two of three). The seizure types included gelastic seizure (three of three) and focal impaired awareness seizure (two of three).

The second pattern (pattern II) showed five patients (Pt. 1, $11,13,14$, and 15) whose cortical hypometabolism was mainly in the lateral temporal cortex. It may also involve the extratemporal cortex, such as the frontal cortex and the parietal cortex, while the mesial temporal cortex is rarely affected (Figure 3). The lateral temporal cortex was involved in all five patients. Of these patients, two (Pt. 11 and 14) had hypometabolism only in the lateral temporal cortex, one (Pt. 1) had hypometabolism in the lateral temporal cortex and the frontal cortex (Figure 3A), and two (Pt. 13 and 15) had hypometabolism in the lateral temporal cortex and the parietal cortex (Figure 3B). The T1-MPRAGE of these patients showed that $\mathrm{HH}$ masses were entirely above the floor of the third ventricle. These HH masses were located in the middle hypothalamus, sparing the mammillary bodies.
A1

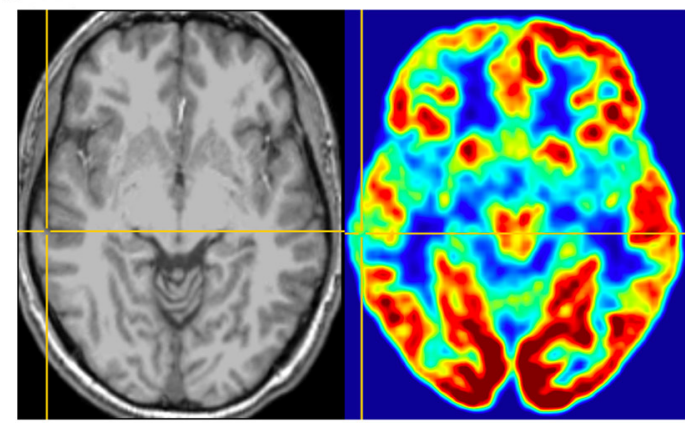

B1

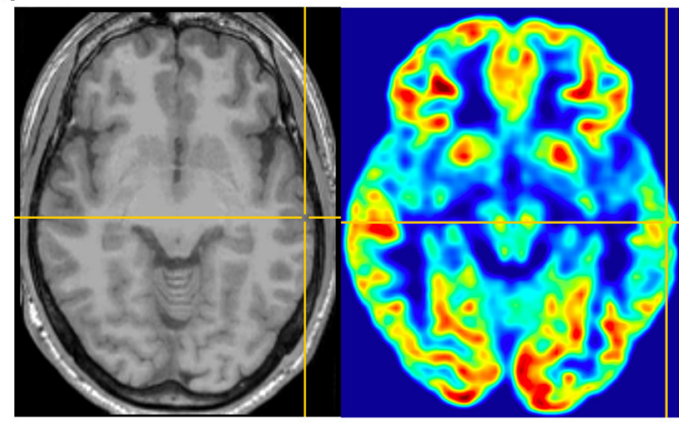

A2

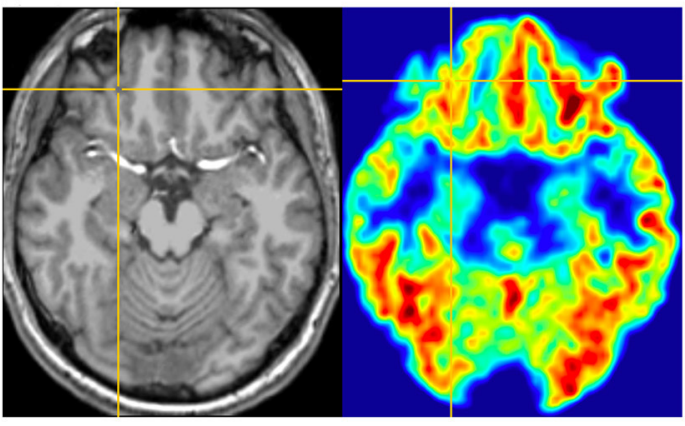

B2

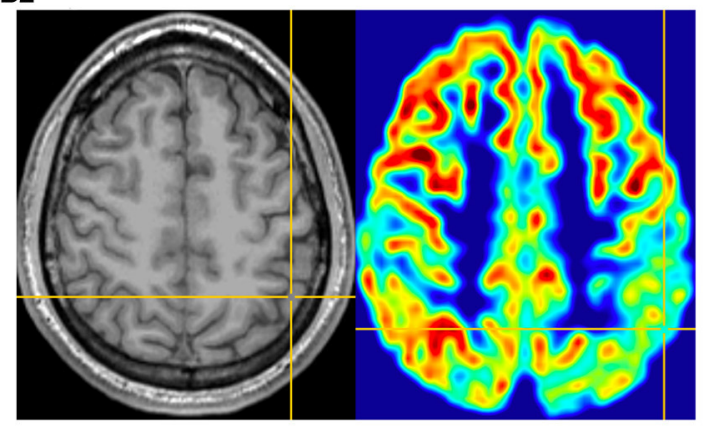

FIGURE 3 | This is the MRI-PET of Pt. 1 (A) and Pt. 13 (B) in the second cortical hypometabolic group. T1-MPRAGE (left) and PET images (right) were co-registered and normalized in $\mathrm{MNI}$ reference. (A) The cortical hypometabolic region is located in right lateral temporal cortex (A1) and right frontal cortex (A2). (B) The cortical hypometabolic region is located in the left lateral temporal cortex (B1) and left parietal cortex (B2). 
There was no interruption on the continuity of the hypersignal in the T1 image, with the structure of the mammillary bodies unchanged (Figure 4). Of the five patients with the second hypometabolic pattern, the main interictal discharge occurred in the frontal-temporal area (three of five) or the temporalparietal area (two of five). The ictal EEG showed right hemisphere paroxysmal fast activity (one of five), no change (two of five), or diffuse electrodecremental events (two of five). Seizure types in this group included gelastic seizure (five of five) and focal to generalized tonic-clonic seizure (four of five).

The third pattern (pattern III) among the other eight patients showed the most extensive range of reduced glucose metabolism in the extra-hypothalamic cortex, including the mesial and lateral temporal, frontal, and parietal cortex (Figure 5). Two patients (Pt. 3 and 16) presented cortical hypometabolism in the mesial temporal cortex, frontal cortex, and parietal cortex (Figure 5A). Two patients (Pt. 2 and 9) presented cortical hypometabolism in the mesial temporal cortex, the lateral temporal cortex, and the parietal cortex (Figure 5B). Four patients (Pt. 5, 6, 8, and 12) presented cortical hypometabolism in the mesial temporal cortex, the lateral temporal cortex, the frontal cortex, and the parietal cortex (Figure 5C). The T1-MPRAGE of the patients showed that $\mathrm{HH}$ masses extended both above and below the third ventricle. The upper portion of the $\mathrm{HH}$ mass was located in the third ventricle, which closely connected with the middle nucleus

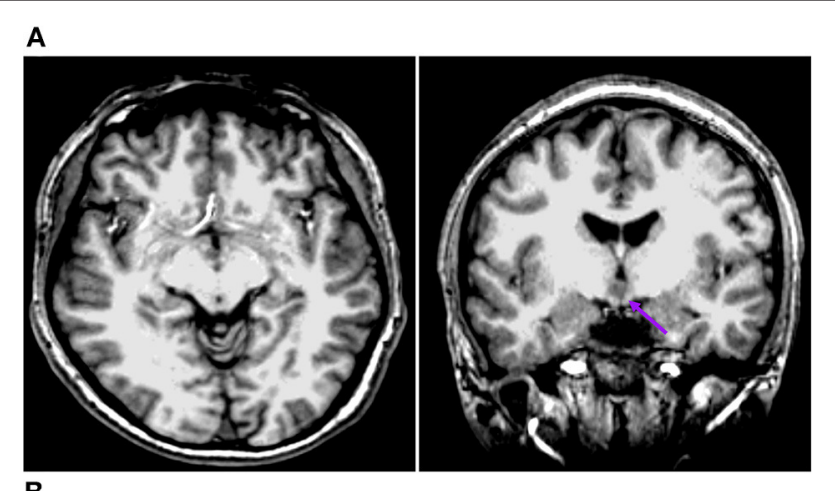

B

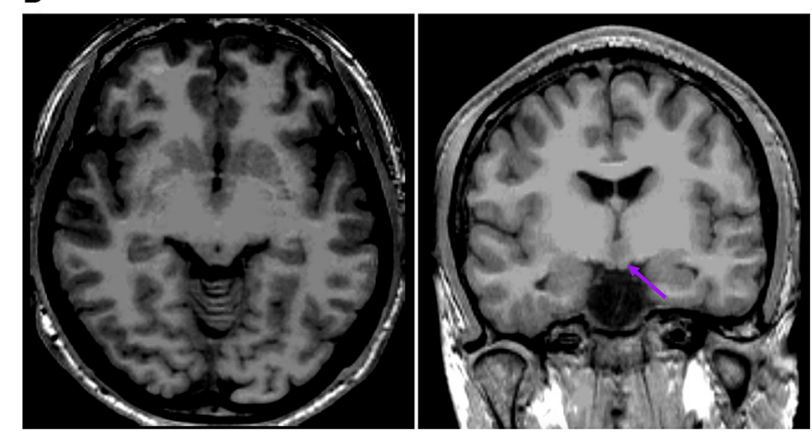

FIGURE 4 | This is the raw T1-MPRAGE images of Pt. 1 (A) and Pt. 13 (B) in the second cortical hypometabolic pattern group. The T1-MPRAGE images (arrows) of $\mathbf{( A )}$ and $\mathbf{( B )}$ showed the mammillary bodies were surrounded by a ring of hypersignal with no interruption and the structure of the mammillary bodies unchanged. The HH mass were entirely above the floor of the third ventricle, tightly connected to the middle nuclei of the hypothalamus. of the hypothalamus. The lower portion was located below the third ventricle, tightly connected with the mammillary bodies (Figure 6). Of the eight patients with the third hypometabolic pattern, the interictal EEG discharge showed temporal spike (one of eight), temporal-parietal spike (one of eight), or diffused spike (six of eight). The ictal EEG discharge showed diffused electrodecremental events or diffused paroxysmal fast activity. The seizure types in this group included gelastic seizure (eight of eight), focal impaired awareness seizure (six of eight), and focal to generalized tonic-clonic seizure (four of eight).

\section{Voxel-Wise FDG-PET Analyses of ROI in Temporal Cortex}

Table 3 presents the mean normalized metabolic voxel values from PET. In the first hypometabolic pattern group, the three patients (Pt. 4, 7, and 10) showed $>10 \%$ asymmetry value in hippocampal formation. No patient showed asymmetry in the amygdala and the lateral temporal neocortex. In the second hypometabolic pattern group, five patients (Pt. 1, 11, 13, 14, and 15 ) showed $>10 \%$ asymmetry value in the lateral temporal neocortex, but no patients showed $>10 \%$ asymmetry value in the hippocampal formation and amygdala. In the third hypometabolic pattern group, $>10 \%$ asymmetry value was found in eight patients within the hippocampal formation, four patients (Pt. 5, 8, 9, and 16) within the amygdala, and five patients (Pt. 2, $6,8,9$, and 12) within the lateral temporal neocortex.

Table 4 presents quantitative analysis of asymmetry value of $\left[{ }^{18} \mathrm{~F}\right]$ FDG PET in ROI of the temporal cortex. In the hypometabolic pattern group I, no statistically significant asymmetry was found between the hippocampal formation (median [IQR]: 1.970 [1.909-2.103] vs. 1.771 [1.720-1.825], $P$ $=0.100$ ), amygdala (median [IQR]: 0.941 [0.869-0.971] vs. 0.881 [0.789-0.889], $P=0.700$ ), and lateral temporal neocortex (median [IQR]: 3.918 [3.622-4.158] vs. 3.720 [3.478-3.741], $P$ $=0.700)$. In the hypometabolic pattern group II, no statistically significant asymmetry was found between the hippocampal formation (median [IQR]: 1.755 [1.682-2.181] vs. 1.673 [1.5832.133], $P=0.310$ ), amygdala (median [IQR]: 0.928 [0.838$1.001]$ vs. 0.830 [0.802-0.953], $P=0.310)$, and lateral temporal neocortex (median [IQR]: 3.452 [3.451-4.518] vs. 3.431 [3.1014.042], $P=0.222$ ). In the hypometabolic pattern group III, the mean normalized PET voxel values were significantly higher in the contralateral hippocampus formations than that in the ipsilateral hippocampus formations (median [IQR]: 1.992 [1.882-2.105] vs. $1.788[1.702-1.824], P=0.001)$; and the mean normalized PET voxel values were significantly higher in the contralateral lateral temporal neocortex than that in the ipsilateral lateral temporal neocortex (median [IQR]: 4.003 [3.783-4.334] vs. $3.672[3.262-3.732], P=0.005)$. No significant differences were found between bilateral amygdale (median [IQR]: 0.891 [0.868-0.970] vs. 0.865 [0.790-0.885], $P=0.083$ ) (Figure 7).

\section{Surgical Prognosis}

The prognosis after surgery of the hypometabolic pattern groups was defined according to the ILAE classification system. A good prognosis corresponded to ILAE classes 1, 2, and 3 (31). Of the 


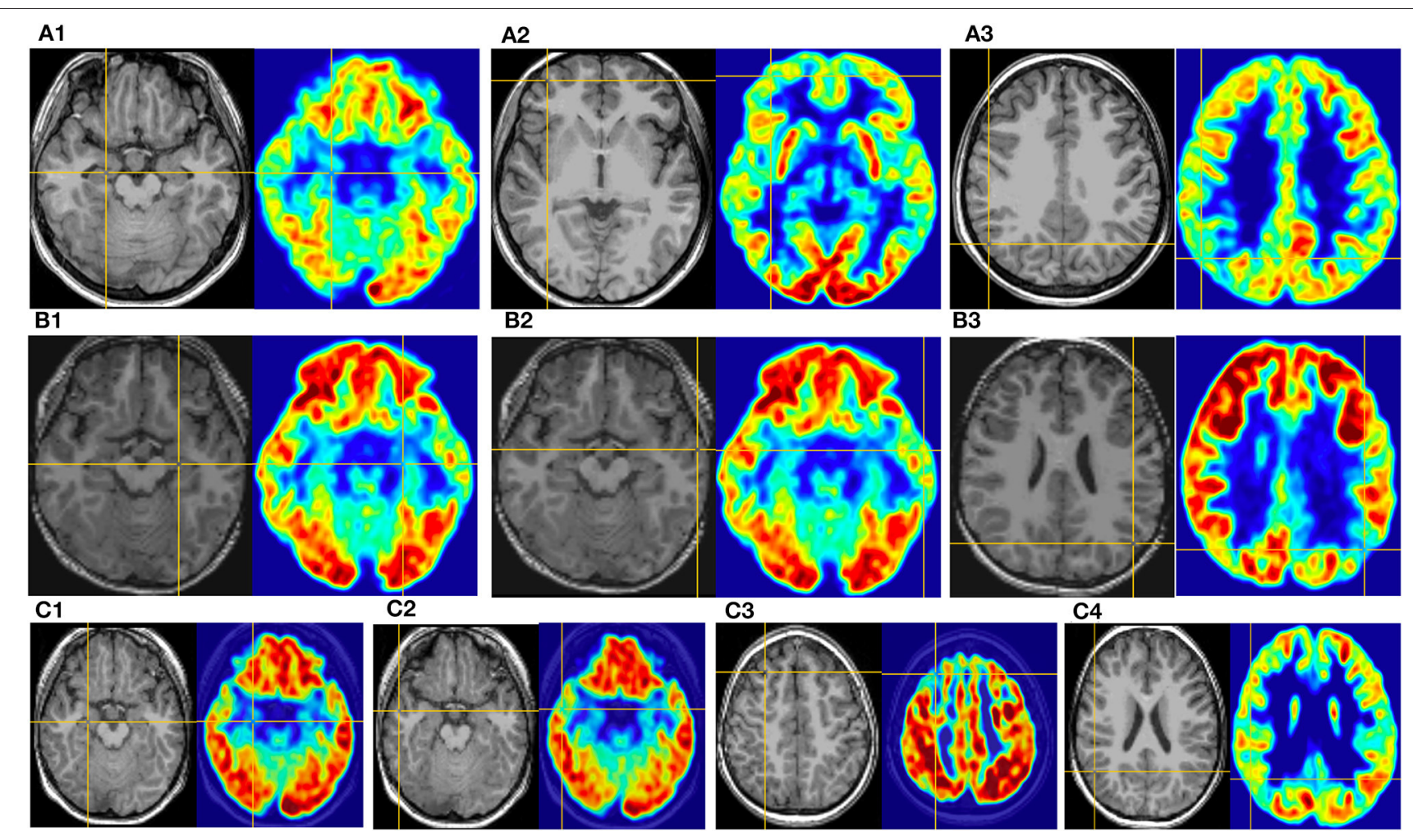

FIGURE 5 | This is the MRI-PET of Pt. 16 (A), Pt. 9 (B), and Pt. 5 (C) in the third cortical hypometabolic pattern group. T1-MPRAGE (left) and PET images (right) were co-registered and normalized in MNI reference. (A) The cortical hypometabolic region is located in the right mesial temporal cortex (A1), right frontal cortex (A2), and right parietal cortex (A3). (B) The cortical hypometabolic region is located in the left mesial temporal cortex (B1), left lateral temporal cortex (B2), and left parietal cortex (B3). (C) The cortical hypometabolic region is located in the right mesial temporal cortex (C1), right lateral temporal cortex (C2), right frontal cortex (C3), and right parietal cortex (C4).

first hypometabolic pattern I, there were two cases (2/3) of ILAE class 1 and one case $(1 / 3)$ of class 4 . For the pattern II, there were two cases of ILAE class $1(2 / 5)$, two cases of class 2 (2/5), and one case of class $4(1 / 5)$. In the pattern III, there were two cases of ILAE class $1(2 / 8)$, one case of class $3(1 / 8)$, four cases of class $4(4 / 8)$, and one case of class $5(1 / 8)$. Hence, the distribution of cases with good prognosis (classes 1-3) was as follows: pattern I $(2 / 3,66.7 \%)$, pattern II $(4 / 5,80 \%)$, and pattern III $(3 / 8,37.5 \%)$. For details, see Table 1.

\section{DISCUSSION}

To our knowledge, this is one study with a relatively large sample size focusing on metabolic distribution characteristics of extra-hypothalamic cortex in patients with $\mathrm{HH}$. With respect to the cortical glucose metabolic characteristics in patients with $\mathrm{HH}$, we found that $\mathrm{HH}$ mass lateralization seen on MRI was significantly correlated with the lateralization of cortical hypometabolism. This is consistent with previous findings. For example, Wagner et al.'s (6) cortical hypometabolism was on the ipsilateral side as the $\mathrm{HH}$ mass in their series. Two independent case reports of PET for $\mathrm{HH}$ showed temporal cortex hypometabolism ipsilateral to $\mathrm{HH}$ mass, consistent with the findings of the scalp and intracranial EEG (7, 32).

In addition, our research found that the hypometabolism pattern of extra-hypothalamic cortex can be divided into three patterns, mainly related to the neuroanatomical location of the $\mathrm{HH}$ mass and was concordant with the cortical regions of the interictal and ictal discharges. In a PET study of five patients with $\mathrm{HH}$, Ryvlin et al. (21) found that the cortical hypometabolic patterns varied greatly among different patients, much like electroclinical findings. These hypometabolic cortexes include the temporal cortex, frontal cortex, and parietal cortex. The findings were consistent with our conclusions. However, Ryvlin's paper may be limited by the insufficient number of cases, and it was impossible to make a specific analysis of the correlation between the hypometabolic distribution pattern of the extrahypothalamus cortex and the neuroanatomic characteristics of the $\mathrm{HH}$ mass. Previous studies have found that $75 \%$ of patients with $\mathrm{HH}$ can develop focal epilepsy with temporal or frontal lobe seizures, depending on the neuroanatomical connection of the $\mathrm{HH}$ mass $(23,24)$. In those studies, cases showing temporal lobe seizures may reportedly be related to the $\mathrm{HH}$ mass invasion of the mammillary bodies, while cases showing frontal lobe seizures may be tightly connected with the middle hypothalamic nuclei (25). Therefore, our paper investigated 


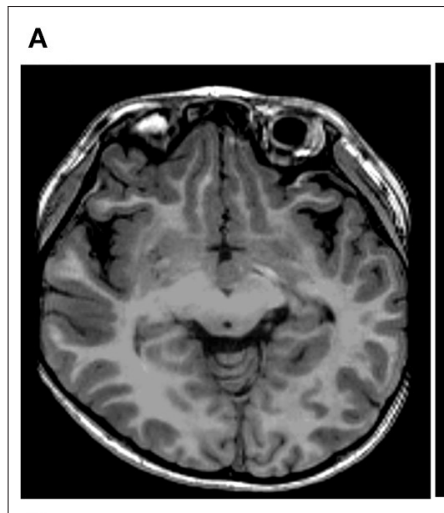

B
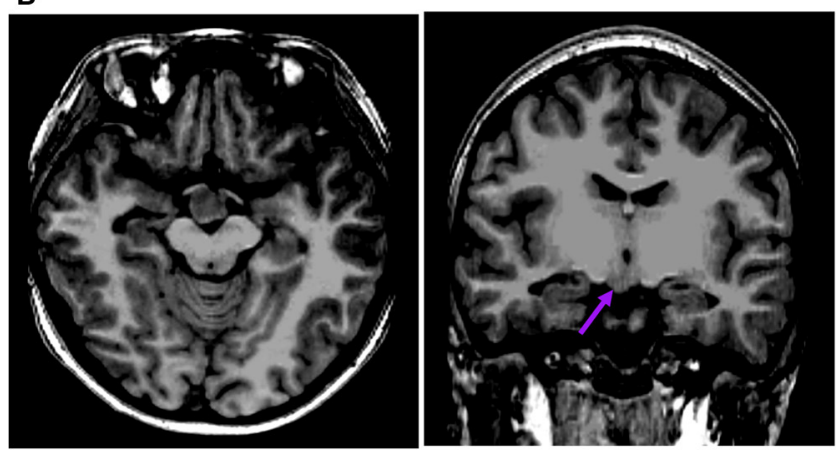

C
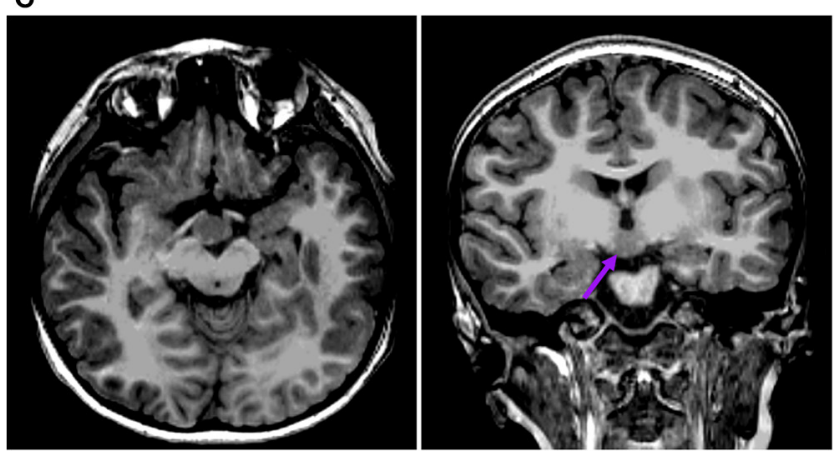

FIGURE 6 | This is the raw T1-MPRAGE images of Pt. 9 (A), Pt. 16 (B), and Pt. 5 (C) in the third cortical hypometabolic pattern group. The T1-MPRAGE images (arrows) of (A-C) showed $\mathrm{HH}$ masses extended both above and below the third ventricle. The upper portion of the $\mathrm{HH}$ mass was located in the third ventricle, which closely connected with the middle nucleus of the hypothalamus. The lower portion was located below the third ventricle, tightly connected with the mammillary bodies.

the correlation between the hypometabolic distribution pattern of extra-hypothalamic cortex and the characteristics of $\mathrm{HH}$ neuroanatomy and electrophysiology.

We found that the first pattern of cortical hypometabolism was in the limbic structures of the mesial temporal lobe. In this group of cases, MRI showed that $\mathrm{HH}$ masses were entirely located below the third ventricle and inside the interpeduncular cistern. The T1 image showed the structure of the mammillary bodies was interrupted and unclear, suggesting that $\mathrm{HH}$ masses may invade the mammillary bodies. Previous literature shows that $\mathrm{HH}$ have a tight connection with the limbic system, such as mesial temporal cortex, through the mammillary bodies (33). List et al. (34) found in the course of autopsy in a patient with $\mathrm{HH}$, a large $\mathrm{HH}$ mass involved with the left mammillary body and showed clinical and EEG characteristics of temporal lobe seizures. Studies have confirmed that the mammillothalamic tract is the most prominent projection of hypothalamus to the cortex (35). The mammillothalamic tract first projected through the mammillary bodies to the anterior nucleus of the thalamus, then to the cingulate gyrus, finally completing the circuit of Papez by projecting back to the mesial temporal cortex. Electrophysiological studies have also confirmed this indirectly (25). In a group of patients with $\mathrm{HH}$ mass connected to the mammillary bodies, the scalp EEG showed a spike in the temporal lobe, especially the mesial temporal cortex. The spike activity might propagate to the subiculum through the fornix, then spread to the mesial temporal cortex throughout the commissural fibers. This may explain the restricted cortical hypometabolism in such patients. The EEG in our group was also mainly manifested as temporal spike, with sphenoid electrode predominantly. The main symptomology included gelastic seizure and focal-impaired awareness seizure. These EEG and clinical manifestation are also consistent with previous results, suggesting that the $\mathrm{HH}$-related seizure can propagate to the mesial temporal cortex. Therefore, these evidences indicate that $\mathrm{HH}$ mass involving mammillary bodies may establish a wide range of anatomical and functional connections to the limbic structures such as the mesial temporal cortex through the mammillothalamic tract, eventually leading to hypometabolism in those regions.

Cortical hypometabolism in the second group of cases was mainly in the lateral temporal cortex, and it can also involve the extratemporal cortex, such as the frontal and parietal cortex. Consistently, MRI in this group showed $\mathrm{HH}$ masses mainly located above the third ventricle, inside the middle hypothalamic nucleus. The $\mathrm{HH}$ masses in this group were mainly attached to the middle nucleus of the hypothalamus, sparing the mammillary bodies. T1 images show the intact mammillary body structure without interruption. Therefore, the hypometabolism of the cortex in this group may be due to the anatomical and functional connection of the $\mathrm{HH}$ masses with the cortex through the middle hypothalamic nucleus. Anterograde tracing studies showed that the middle hypothalamic neurons project through the medial forebrain bundle into the cerebral cortex. At the preoptic area, fibers run ventrally to globus pallidus, putamen, and external capsule, from which they project to much of the lateral temporal cortex outside the hippocampal formation (35). A DTI study also identified that hypothalamic neurons also connected to the parietal and occipital regions through the temporooccipital fascicle (36). Connections to the frontal cortex were also found (6). The EEG in our group was mainly manifested as frontotemporal or temporalparietal discharge. The main symptomology included gelastic seizure and focal to generalized tonic-clonic seizures. This is grossly concordant with previous studies, suggesting the $\mathrm{HH}$ mass involving the middle hypothalamic nucleus may have connections to the lateral temporal cortex and/or extratemporal 
TABLE 3 | Quantitative analysis of [18F] FDG PET of ROI in temporal cortex.

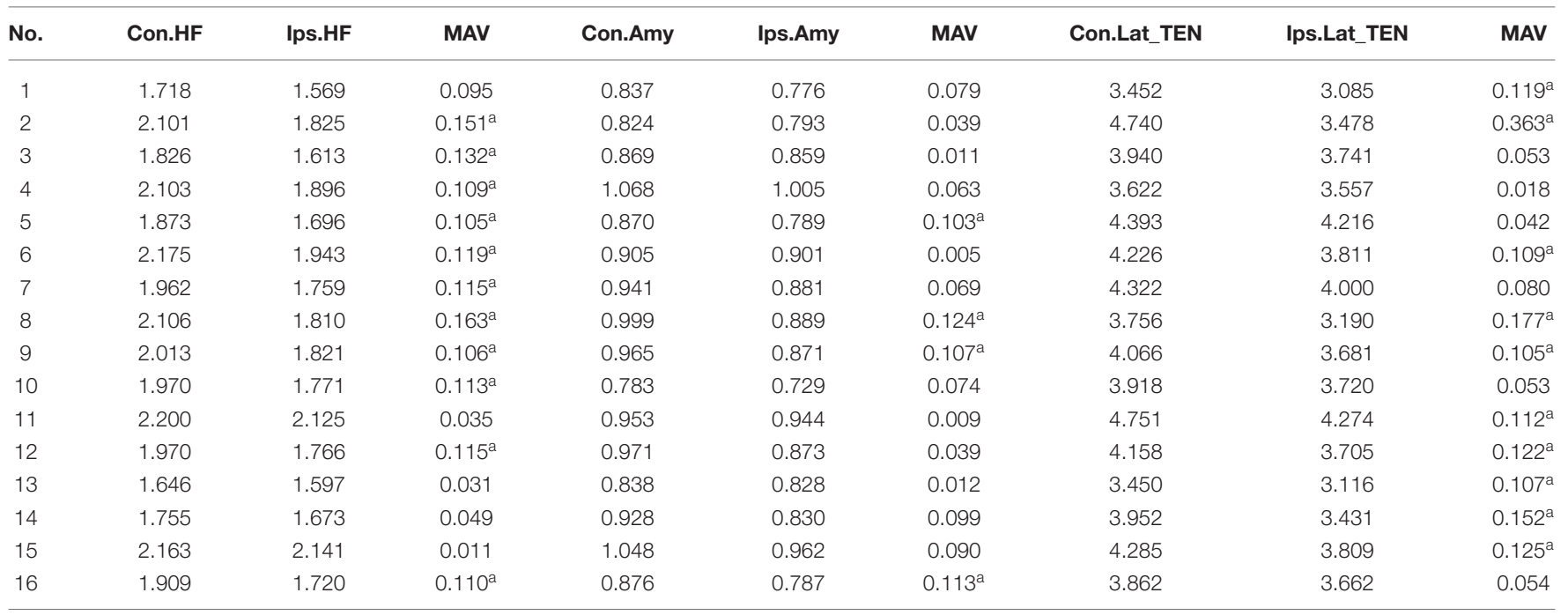

Con, contralateral; Ips ipsilateral; HF, hippocampal formation; MAV, metabolism asymmetric value; Amy, amygdala; Lat_TEN, lateral temporal neocortex, a > 10\%.

TABLE 4 | Asymmetry measurements of PET voxel value of the temporal cortex of three hypometabolic patterns.

\begin{tabular}{|c|c|c|c|c|c|c|}
\hline & Con.HF & Ips.HF & Con.Amy & Ips.Amy & $\begin{array}{l}\text { Con.Lat } \\
\text { TEN }\end{array}$ & $\begin{array}{l}\text { Ips.Lat } \\
\text { TEN }\end{array}$ \\
\hline \multicolumn{7}{|l|}{ PATTERN 1} \\
\hline$P$-value & \multicolumn{2}{|c|}{0.100} & \multicolumn{2}{|c|}{0.700} & \multicolumn{2}{|c|}{0.700} \\
\hline$P$-value & \multicolumn{2}{|c|}{0.310} & \multicolumn{2}{|c|}{0.310} & \multicolumn{2}{|c|}{0.222} \\
\hline \multicolumn{7}{|l|}{ PATTERN 3} \\
\hline $\begin{array}{l}\text { Median } \\
\text { [IQPR] }\end{array}$ & $\begin{array}{c}1.992 \\
{[1.882-2.105]}\end{array}$ & $\begin{array}{c}1.788 \\
{[1.702-1.824]}\end{array}$ & $\begin{array}{c}0.891 \\
{[0.868-0.970]}\end{array}$ & $\begin{array}{c}0.865 \\
{[0.790-0.885]}\end{array}$ & $\begin{array}{c}4.112 \\
{[3.881-4.351]}\end{array}$ & $\begin{array}{c}3.693 \\
{[3.524-3.794]}\end{array}$ \\
\hline$P$-value & \multicolumn{2}{|c|}{0.001} & \multicolumn{2}{|c|}{0.083} & \multicolumn{2}{|c|}{0.005} \\
\hline
\end{tabular}

Con, contralateral; Ips, ipsilateral; HF, hippocampal formation; Amy, amygdala; Lat_TEN, lateral; IQR, interquartile range.

(frontal or parietal) cortex, leading to hypometabolism in those regions.

The third group of cases has the widest range of cortical hypometabolic regions, including the mesial and lateral temporal cortex, frontal cortex, and parietal cortex. T1-MPRAGE of this group showed that the $\mathrm{HH}$ mass located both above and below the third ventricle, with part of the $\mathrm{HH}$ mass inside the third ventricle and with part across the floor of the third ventricle, entering the interpeduncular cisterna. The portion of the $\mathrm{HH}$ mass below the third ventricle may affect the mammillary bodies, while the portion of the $\mathrm{HH}$ mass above the third ventricle may involve the middle nucleus of the hypothalamus. The $\mathrm{HH}$ mass in this group can connect to extrahypothalamic cortex through both pathways described in the first and second groups. Therefore, the cortical hypometabolic region of this group was the most extensive, and the cortex of EEG discharge was diffused, with weak localization values. The patient's seizure patterns were the most diverse, with gelastic seizure, focal impaired awareness seizure, and focal to generalized tonic-clonic seizures. Generalized tonic-clonic seizures showed the highest frequency in this group of patients. The prognosis of patients with the third type was the worst, which is also consistent with previous literature reports indicating that the higher the extension of the hypometabolism in cortex, the worst is the prognosis after surgery. The previous study showed the prognosis of those with hypometabolism in the mesial temporal lobe is better than that of those with hypometabolism in cortex other than the mesial temporal lobe among patients with mesial temporal epilepsy (37). This point also shows that the study of PET in $\mathrm{HH}$ patients is of great significance in guiding the preoperative evaluation of $\mathrm{HH}$ patients and judging the prognosis.

We believe that our study was an initial step toward metabolic characterization of patients with $\mathrm{HH}$ according to the clinical 


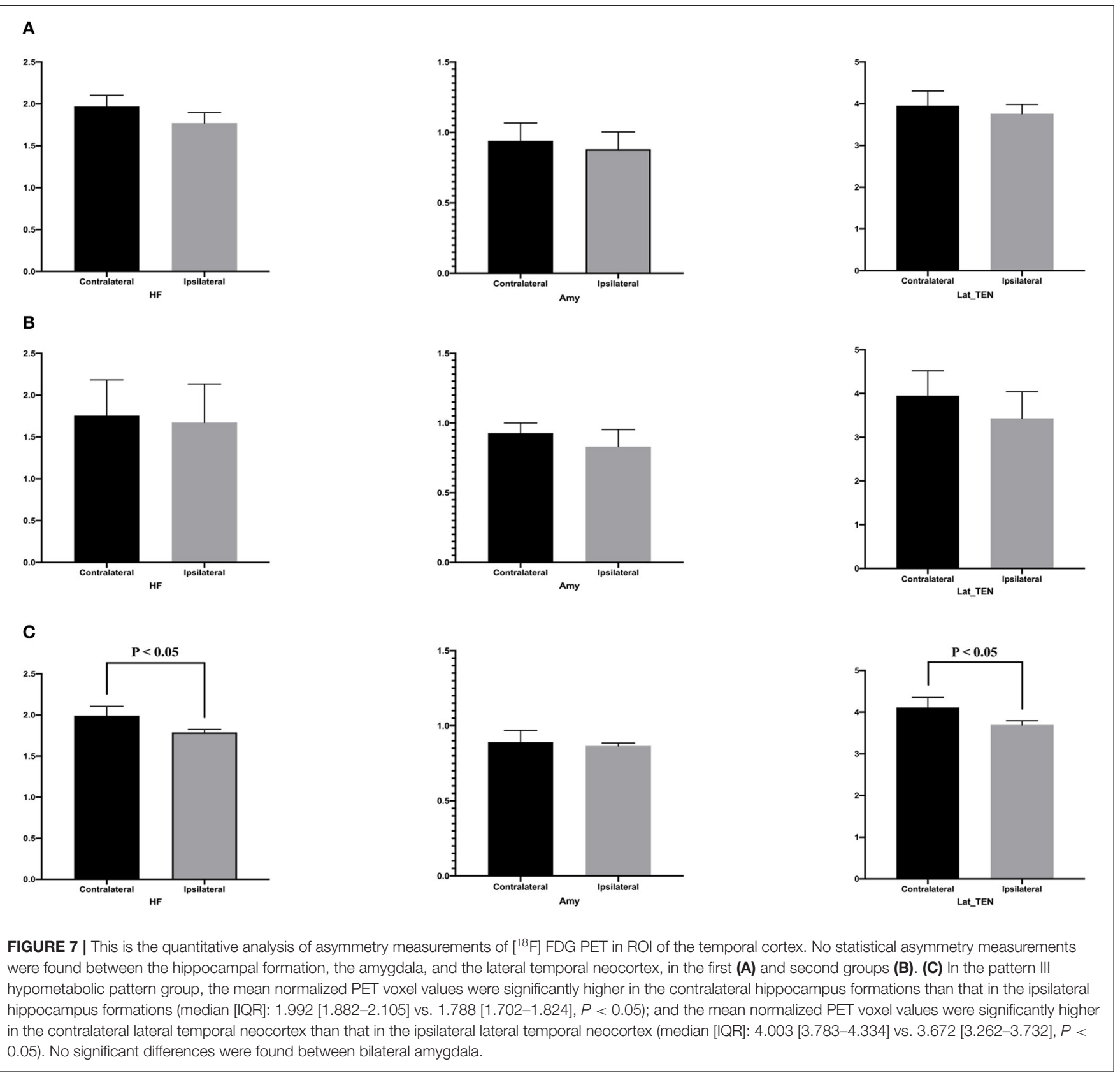

question that the single epileptogenic hamartoma might lead to cortical abnormality outside $\mathrm{HH}$ during the disease process. In our patients, the fact that the lateralization of hypometabolism always occurred ipsilateral in MRI and EEG findings reinforced the view that the ipsilateral extra-hypothalamic cortical area is affected during the disease process. As for the hypometabolism pattern of extra-hypothalamic cortex, we combined visual analysis with quantification of the asymmetry value because of the difficulty to obtain normative FDG data from matched healthy control subjects to compare with the affected patients, especially for children. The results demonstrated that, in the third group, differences were found between the contralateral and ispilateral sides of the hippocampal formation and the lateral temporal neocortex. By contrast, no statistically significant asymmetry was found between the hippocampal formations, or in the lateral temporal neocortex, in the first and second groups, respectively. Lack of asymmetry value in these groups may be a result of an insufficient sample size because significantly hypometabolic regions were present with visual rating in these patients. In the future, we will continue to enroll more cases to better characterize glucose metabolism associated with $\mathrm{HH}$ neuroanatomy and therein further elucidate the complex pathophysiology of aberrant connection with remote cortex in patients with $\mathrm{HH}$. 
Several limitations of our study must be pointed out. First, because PET is an invasive examination, PET data from a group of healthy individuals was difficult to be obtained, and there were no such data retained in the early stage. The asymmetry assessment method used in the paper ignored potential agerelated changes in normal metabolic asymmetries and could not address potential bilateral hypometabolism in these regions. Second, EEG was not monitored during the tracer uptake period; thus, subclinical seizures or frequent interictal epileptiform activity might affect metabolism. Third, due to the small sample data and limited statistical analysis, the study was an initial step to suggest three hypometabolic patterns in patients with $\mathrm{HH}$ and the third pattern may be associated with poor prognosis; thus, more studies are needed to confirm these data in the future.

\section{DATA AVAILABILITY STATEMENT}

The original contributions presented in the study are included in the article/supplementary material, further inquiries can be directed to the corresponding author/s.

\section{ETHICS STATEMENT}

The studies involving human participants were reviewed and approved by Ethic committee of Xuanwu Hospital of Capital

\section{REFERENCES}

1. Berkovic SF, Andermann F, Melanson D, Ethier RE, Feindel W, Gloor P. Hypothalamic hamartomas and ictal laughter: evolution of a characteristic epileptic syndrome and diagnostic value of magnetic resonance imaging. Ann Neurol. (1988) 23:429-39. doi: 10.1002/ana.410230502

2. Kerrigan JF, Ng YT, Chung S, Rekate HL. The hypothalamic hamartoma: a model of subcortical epileptogenesis and encephalopathy. Semin Pediatr Neurol. (2005) 12:119-31. doi: 10.1016/j.spen.2005.04.002

3. Prigatano GP. Cognitive and behavioral dysfunction in children with hypothalamic hamartoma and epilepsy. Semin Pediatr Neurol. (2007) 14:6572. doi: 10.1016/j.spen.2007.03.004

4. Striano S, Striano P, Coppola A, Romanelli P. The syndrome gelastic seizureshypothalamic hamartoma: severe, potentially reversible encephalopathy. Epilepsia. (2009) 50 (Suppl. 5):62-5. doi: 10.1111/j.1528-1167.2009.02125.x

5. Sonoda M, Masuda H, Shirozu H, Ito Y, Akazawa K, Asano E. Predictors of cognitive function in patients with hypothalamic hamartoma following stereotactic radiofrequency thermocoagulation Surgery. (2017) 58:155665. doi: 10.1111/epi.13838

6. Wagner K, Schulze-Bonhage A, Urbach H, Trippel M, Spehl TS, Buschmann F, et al. Reduced glucose metabolism in neocortical network nodes remote from hypothalamic hamartomas reflects cognitive impairment. Epilepsia. (2017) 58 (Suppl. 2):41-9. doi: 10.1111/epi.13757

7. Cascino GD, Andermann F, Berkovic SF, Kuzniecky RI, Sharbrough FW, Keene DL, et al. Gelastic seizures and hypothalamic hamartomas: evaluation of patients undergoing chronic intracranial EEG monitoring and outcome of surgical treatment. Neurology. (1993) 43:747-50. doi: 10.1212/WNL.43.4.747

8. Munari C, Kahane P, Francione S, Hoffmann D, Tassi L, Cusmai R, et al. Role of the hypothalamic hamartoma in the genesis of gelastic fits (a videostereo-EEG study). Electroencephalogr Clin Neurophysiol. (1995) 95:15460. doi: 10.1016/0013-4694(95)00063-5

9. Fenoglio KA, Wu J, Kim DY, Simeone TA, Coons SW, Rekate H, et al. Hypothalamic hamartoma: basic mechanisms of intrinsic epileptogenesis. Semin Pediatr Neurol. (2007) 14:51-9. doi: 10.1016/j.spen.2007.03.002
Medical University, Beijing, China. Written informed consent to participate in this study was provided by the participants' legal guardian/next of kin. Written informed consent was obtained from the individual(s), and minor(s)' legal guardian/next of kin, for the publication of any potentially identifiable images or data included in this article.

\section{AUTHOR CONTRIBUTIONS}

Y-FY contributed to the data acquisition and analysis of the manuscript. Y-ZS and G-GZ contributed to the data acquisition, analysis and redaction of the manuscript, and also the interpretation of the data. P-HW, Y-HW, and L-KR contributed to the data acquisition and analysis. YA, FM, and DW contributed to data acquisition. All authors read and approved the final manuscript.

\section{FUNDING}

This work was supported by the National Key Research and Development Program of China (Grant No. 2016YFC0103909) and National Natural Science Foundation of China (Grant No. 81871009).
10. Steinmetz PN, Wait SD, Lekovic GP, Rekate HL, Kerrigan JF. Firing behavior and network activity of single neurons in human epileptic hypothalamic hamartoma. Front Neurol. (2013) 4:210. doi: 10.3389/fneur.2013.00210

11. Kerrigan JF, Parsons A, Tsang C, Simeone K, Coons S, Wu J. Hypothalamic hamartoma: neuropathology and epileptogenesis. Epilepsia. (2017) 58 (Suppl.)2:22-31. doi: 10.1111/epi.13752

12. Scholly J, Staack AM, Kahane P, Scavarda D, Regis J, Hirsch E, et al. Hypothalamic hamartoma: epileptogenesis beyond the lesion? Epilepsia. (2017) 58 (Suppl.2):32-40. doi: 10.1111/epi.13755

13. Bartolomei F, Guye M, Wendling F. Abnormal binding and disruption in large scale networks involved in human partial seizures. EPJ Nonlinear Biomedical Physics. (2013) 1:4. doi: 10.1140/epjnbp11

14. Kahane P, Ryvlin P, Hoffmann D, Minotti L, Benabid AL. From hypothalamic hamartoma to cortex: what can be learnt from depth recordings and stimulation? Epileptic Disord. (2003) 5:205-17.

15. Goddard GV. Development of epileptic seizures through brain stimulation at low intensity. Nature. (1967) 214:1020-1. doi: 10.1038/2141020a0

16. Mauguière F, Ryvlin P. The role of PET in presurgical assessment of partial epilepsies. Epileptic Disord. (2004) 6:193-215.

17. Castro LH, Ferreira LK, Teles LR, Jorge CL, Arantes PR, Ono CR, et al. Epilepsy syndromes associated with hypothalamic hamartomas. Seizure. (2007) 16:50-8. doi: 10.1016/j.seizure.2006.10.008

18. Kameyama S, Shirozu H, Masuda H, Ito Y, Sonoda M, Akazawa K. MRIguided stereotactic radiofrequency thermocoagulation for 100 hypothalamic hamartomas. J Neurosurg. (2016) 124:1503-12. doi: 10.3171/2015.4. JNS1582

19. Killeen Z, Bunch R, Kerrigan JF. Psychiatric comorbidity with hypothalamic hamartoma: systematic review for predictive clinical features. Epilepsy Behav. (2017) 73:126-30. doi: 10.1016/j.yebeh.2017. 05.019

20. Gaillard WD, Fazilat S, White S, Malow B, Sato S, Reeves P, et al. Interictal metabolism and blood flow are uncoupled in temporal lobe cortex of patients with complex partial epilepsy. Neurology. (1995) 45:18417. doi: 10.1212/WNL.45.10.1841 
21. Ryvlin P, Ravier C, Bouvard S, Mauguire F, Le Bars D, Arzimanoglou A, et al. Positron emission tomography in epileptogenic hypothalamic hamartomas. Epileptic Disord. (2003) 5:219-27.

22. Parvizi J, Le S, Foster BL, Bourgeois B, Riviello JJ, Prenger E, et al. Gelastic epilepsy and hypothalamic hamartomas: neuroanatomical analysis of brain lesions in 100 patients. Brain. (2011) 134:2960-8. doi: 10.1093/brain/awr235

23. Mullatti N, Selway R, Nashef L, Elwes R, Honavar M, Chandler $\mathrm{C}$, et al. The clinical spectrum of epilepsy in children and adults with hypothalamic hamartoma. Epilepsia. (2003) 44:13109. doi: 10.1046/j.1528-1157.2003.04103.x

24. Oehl B, Brandt A, Fauser S, Bast T, Trippel M, Schulze-Bonhage A. Semiologic aspects of epileptic seizures in 31 patients with hypothalamic hamartoma. Epilepsia. (2010) 51:2116-23. doi: 10.1111/j.1528-1167.2010. 02686.x

25. Leal AJ, Moreira A, Robalo C, Ribeiro C. Different electroclinical manifestations of the epilepsy associated with hamartomas connecting to the middle or posterior hypothalamus. Epilepsia. (2003) 44:11915. doi: 10.1046/j.1528-1157.2003.66902.x

26. Delalande O, Fohlen M. Disconnecting surgical treatment of hypothalamic hamartoma in children and adults with refractory epilepsy and proposal of a new classification. Neurol Med Chir (Tokyo). (2003) 43:61-8. doi: 10.2176/nmc.43.61

27. Luders H, Acharya J, Baumgartner C, Benbadis S, Bleasel A, Burgess R, et al. Semiological seizure classification. Epilepsia. (1998) 39:100613. doi: 10.1111/j.1528-1157.1998.tb01452.x

28. Lamarche F, Job AS, Deman P, Bhattacharjee M, Hoffmann D, GallazziniCrepin C, et al. Correlation of FDG-PET hypometabolism and SEEG epileptogenicity mapping in patients with drug-resistant focal epilepsy. Epilepsia. (2016) 57:2045-55. doi: 10.1111/epi.13592

29. Pustina D, Avants B, Sperling M, Gorniak R, He X, Doucet G, et al. Predicting the laterality of temporal lobe epilepsy from PET, MRI, and DTI: a multimodal study. Neuroimage Clin. (2015) 9:20-31. doi: 10.1016/j.nicl.2015.07.010

30. Jokeit H, Seitz RJ, Markowitsch HJ, Neumann N, Witte OW, Ebner A. Prefrontal asymmetric interictal glucose hypometabolism and cognitive impairment in patients with temporal lobe epilepsy. Brain. (1997) 120:228394. doi: 10.1093/brain/120.12.2283

31. Wieser HG, Blume WT, Fish D, Goldensohn E, Hufnagel A, King D, et al. ILAE Commission Report. Proposal for a new classification of outcome with respect to epileptic seizures following epilepsy surgery. Epilepsia. (2001) 42:282-6. doi: 10.1046/j.1528-1157.2001.4220282.x

32. Meyer MA. Temporal lobe hypometabolism ipsilateral to a hypothalamic mass. Relationship to Gelastic Seizures Clin Positron Imaging. (2000) 3:757. doi: 10.1016/S1095-0397(00)00043-1

33. Vanderah T, Gould DJ. Nolte's The Human Brain E-Book: An Introduction to its Functional Anatomy. Elsevier Health Sciences (2015).

34. List CF, Dowman CE, Bagchi BK, Bebin J. Posterior hypothalamic hamartomas and gangliogliomas causing precocious puberty. Neurology. (1958) 8:164. doi: 10.1212/WNL.8.3.164

35. Mai JK, Paxinos G. The Human Nervous System. Academic Press (2011).

36. Lemaire JJ, Frew AJ, Mcarthur D, Gorgulho AA, Alger JR, Salomon N, et al. White matter connectivity of human hypothalamus. Brain Res. (2011) 1371:43-64. doi: 10.1016/j.brainres.2010.11.072

37. Salanova V, Markand O, Worth R, Smith R, Wellman H, Hutchins G, et al. FDG-PET and MRI in temporal lobe epilepsy: relationship to febrile seizures, hippocampal sclerosis and outcome. Acta Neurol Scand. (1998) 97:146-53. doi: 10.1111/j.1600-0404.1998.tb00628.x

Conflict of Interest: The authors declare that the research was conducted in the absence of any commercial or financial relationships that could be construed as a potential conflict of interest.

Copyright (c) 2021 Yang, Wei, Meng, An, Fan, Wang, Wang, Ren, Shan and Zhao. This is an open-access article distributed under the terms of the Creative Commons Attribution License (CC BY). The use, distribution or reproduction in other forums is permitted, provided the original author(s) and the copyright owner(s) are credited and that the original publication in this journal is cited, in accordance with accepted academic practice. No use, distribution or reproduction is permitted which does not comply with these terms. 